\title{
Avaliação da adesão à prevenção de doença cardiovascular em usuários da estratégia de saúde da família
}

\author{
Assessment of adherence to prevention of cardiovascular disease in users of the family health \\ strategy \\ Evaluación de la adherencia a la prevención de enfermedades cardiovasculares em usuarios de la \\ estrategia de salud familiar
}

Recebido: 03/06/2021 | Revisado: 09/06/2021 | Aceito: 11/06/2021 | Publicado: 26/06/2021

\author{
Milena Santana Ribeiro e Silva \\ ORCID: https://orcid.org/0000-0002-0196-574X \\ Universidade Tiradentes, Brasil \\ E-mail: milena_ris@ hotmail.com \\ Úrsula Maria Moreira Costa Burgos \\ ORCID: https://orcid.org/0000-0001-7234-4046 \\ Universidade Tiradentes, Brasil \\ E-mail: ursulacostab@gmail.com
}

\begin{abstract}
Resumo
A terapêutica para controle de doenças cardiovasculares (DCV) segue os parâmetros propostos em saúde cardiovascular (SCV) pela American Heart association (AHA) que inclui métricas comportamentais: tabagismo, dieta e atividade física e métricas biológicas compostas por IMC, pressão arterial, colesterol e glicemia sérica. O monitoramento dessas métricas se associa a redução de mortes e hospitalizações por DCV. Com o mesmo intuito, no Brasil, a estratégia de saúde da família (ESF) promove reuniões mensais com ações educativas, incentivo à prática de exercícios físicos, consultas médicas agendadas e entrega de medicamentos na atenção básica. Este estudo visa, por meio de uma revisão integrativa, discutir a respeito das dificuldades existentes no controle de doenças cardiovasculares (DCV), considerando o aspecto multifatorial do processo de prevenção e tratamento.
\end{abstract}

Palavras-chave: Adesão; Doença cardiovascular; Atenção básica; Tratamento; Prevenção.

\begin{abstract}
Cardiovascular disease (CVD) management therapy follows the parameters proposed in cardiovascular health (CVS) by the American Heart association (AHA) which includes behavioral metrics: smoking, diet and physical activity and biological metrics composed of BMI, blood pressure, serum cholesterol and blood glucose. Monitoring these metrics is associated with a reduction in CVD deaths and hospitalizations. With the same purpose, in Brazil, the family health strategy (ESF) promotes monthly meetings with educational actions, encouragement to practice physical exercises, scheduled medical appointments, and delivery of medicines in primary care. This study aims, through an integrative review, to discuss the existing difficulties in the control of cardiovascular diseases (CVD), considering the multifactorial aspect of the process of prevention and treatment.
\end{abstract}

Keywords: Adherence; Cardiovascular disease; Primary care; Treatment; Prevention.

\section{Resumen}

La terapia de control de las enfermedades cardiovasculares (ECV) sigue los parámetros propuestos en materia de salud cardiovascular (CVS) por la Asociación Americana del Corazón (AHA), que incluye métricas conductuales: tabaquismo, dieta y actividad física y métricas biológicas compuestas por el IMC, la presión arterial, el colesterol sérico y la glucemia. El seguimiento de estas métricas se asocia a una reducción de las muertes y hospitalizaciones por ECV. Con la misma intención, en Brasil, la estrategia de salud familiar (ESF) promueve reuniones mensuales con acciones educativas, incentivo a la práctica de ejercicios físicos, citas médicas programadas y entrega de medicamentos en la atención primaria. Este estudio pretende, mediante una revisión integradora, discutir las dificultades existentes en el control de las enfermedades cardiovasculares (ECV), considerando el aspecto multifactorial del proceso de prevención y tratamiento.

Palabras clave: Adherencia; Enfermedad cardiovascular; Atención primaria; Tratamiento; Prevención.

\section{Introdução}

O tratamento preventivo de doenças cardiovasculares (DCV) inclui o acesso medicamentoso e sua correta forma de administração, os cuidados alimentares e a prática de exercícios físicos para fins de controle de índice de massa corpórea 
(IMC) e dos níveis de colesterol, por exemplo. É nítido que a adesão à profilaxia de patologias cardiovasculares envolve aspectos da vida de seus pacientes e abrange desde os recursos disponíveis até maneira com a qual a prevenção é manejada pelos profissionais de saúde.

A adesão terapêutica perpassa pela orientação dos profissionais de saúde, pelos métodos utilizados para reforçá-las, mas, principalmente, pelo cotidiano do paciente, relacionando-se intimamente ao seu estilo de vida, comportamento, costumes, prazeres, partilhas, aprendizados, noções e percepção do próprio corpo e saúde. Tal relação age não somente na integração mas também na modificação da vida e rotina do paciente nos aspectos aqui mencionados.

Este estudo objetiva contribuir com a estratégia de saúde da família (ESF) como atuante no controle das DCV, analisando os fatores de risco no nosso meio e identificando na condução das práticas da ESF quais são as maiores dificuldades no processo de adesão terapêutica, incluindo farmacológica e não farmacológica.

\section{Metodologia}

Este estudo qualitativo revisou integralmente vinte referências, incluindo publicações periódicas e artigos, pesquisados nas bases de dados PUBMED e SCHOLAR GOOGLE. Foram empregadas as palavras-chave adesão, doença cardiovascular, atenção básica, tratamento e prevenção, em português, usadas isoladamente e em combinação.

Inicialmente foram filtrados os materiais a respeito dos tratamentos medicamentosos para Hipertensão Arterial Sistêmica (HAS), Diabetes Melitus (DM) e dislipidemia na atenção básica, nesta ordem de busca, e escolhidos os artigos contendo maior abrangência de dados das regiões brasileiras sobre os medicamentos mais utilizados e distribuição do recurso em tais áreas do país. Foram excluídas as leituras restritas a um estado ou Unidade de Saúde apenas, abstidas de material comparativo entre diferentes pontos do Brasil ou que não pudesse embasar essa análise. A seleção acerca do tratamento não farmacológico considerou artigos dos últimos 5 anos com os quesitos alimentação, tabagismo e exercício físico com maior riqueza descritiva direcionada à adesão terapêutica e excluiu materiais sem esse foco.

Da apuração de 30 materiais, foram escolhidos 10 na primeira plataforma de pesquisa e 10 na segunda base de dados, ncluindo artigos, livros e publicações periódicas especializadas; Foram excluídos 10 materiais por não serem compatíveis com a proposta da presente revisão. O fluxograma abaixo sintetiza o processo de seleção de materiais aqui colocada.

Figura 1 - Processo de seleção dos materiais para a pesquisa.

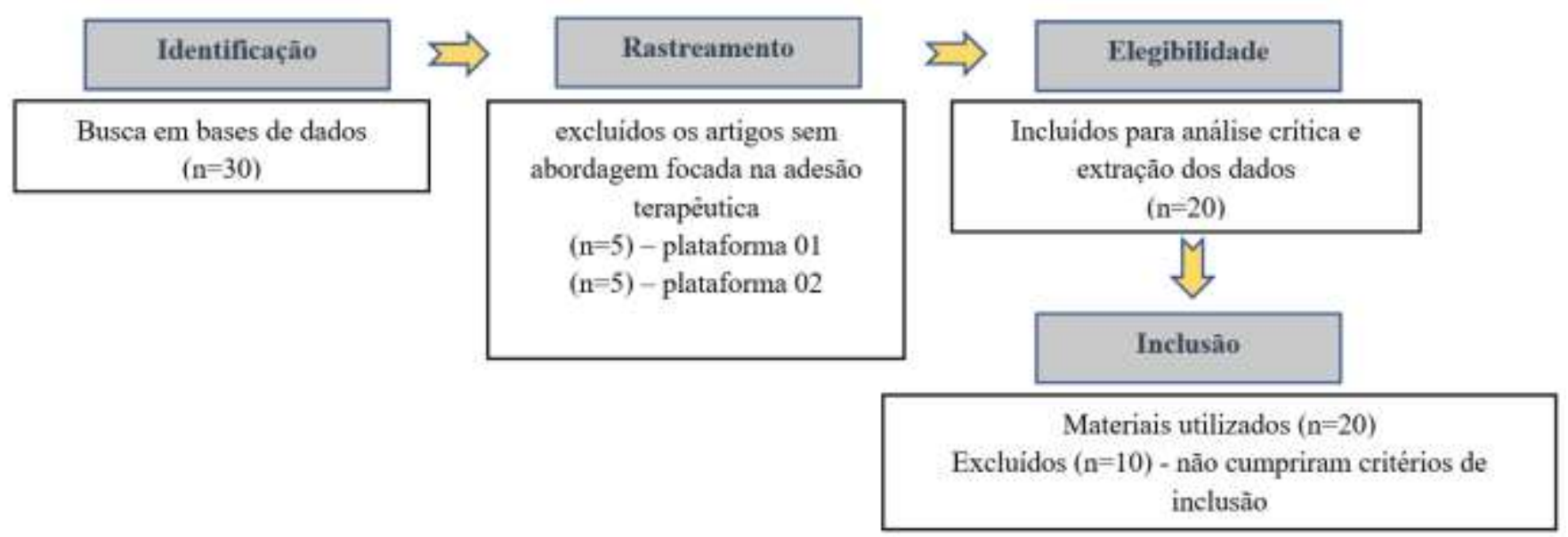

Fonte: Autores. 


\section{Resultados e Discussão}

Ainda é diminuta a quantidade de análises acuradas acerca do que influencia favoravelmente ou não a adesão a tratamentos. Pretendendo ampliar esse acervo, esta discussão analisa os estudos disponíveis sobre adesão às terapêuticas medicamentosa e não medicamentosa preventivas de DCV, expondo quesitos preponderantes ao seguimento das recomendações médicas. A PNAUM (Pesquisa Nacional sobre Acesso, Utilização e Promoção do Uso Racional de Medicamentos), em 2014, associou a aderência do paciente ao tratamento medicamentoso para doenças crônicas a fatores demográficos, socioeconômicos, atenção e percepção à saúde, e uso de medicamentos. Constatou-se que a baixa adesão é mais comum nos seguintes grupos: adultos jovens, de baixa escolaridade, das regiões nordeste e Centro-Oeste do Brasil, pacientes que precisaram arcar com o custo do tratamento, os que tem uma autopercepção ruim da saúde, os acometidos com três ou mais doenças e os limitados por alguma das doenças crônicas (Mengue SS et al, 2014).

\subsection{Terapêutica farmacológica}

As doenças crônicas não transmissíveis (DCNT) são um problema global de saúde por apresentarem grande mortalidade e custo com hospitalizações, e estão diretamente relacionadas a DCV. A OMS considera o acesso medicamentoso como parte essencial para alcançar a meta de diminuição de mortalidade por DCNT. (Mengue SS et al, 2014). Entre estas, a responsável pelo maior número de doentes é a hipertensão arterial sistêmica (HAS). Desse modo, é possível analisar o controle da doença cardiovascular por meio do uso correto ou não de anti-hipertensivos.

\subsubsection{Hipertensão arterial}

Os fármacos específicos mais utilizados - isoladamente - em ordem decrescente são: Hidroclorotiazida, Losartana, Captopril, Enalapril, Atenolol e Anlodipino. Essas medicações estão no grupo das definitivamente usadas para tratar HAS, em sua maioria como monofármacos e minoritariamente associados em doses fixas. Dessas associações, a mais comum é Hidroclorotiazida + Losartana, Atenolol ou Amilorida. Esses medicamentos são acessados com menor custo nos estágios iniciais da doença. (Mengue SS et al, 2014).

Certamente, com o avanço da patologia, tem-se o aumento no gasto com a medicação gerando fator financeiro preponderante no tratamento, quando há necessidade de o paciente arcar com os remédios. A iniciativa de projetos públicos como farmácia popular são intervenções positivas que amplificam o acesso medicamentoso à população. A dispensação dos remédios pelas farmácias nos postos de ESF tem participação ativa no controle de doenças e portanto a descontinuidade desse serviço de fornecimento ou queda na qualidade dos medicamentos podem agravar as patologias em questão e gerar mais gastos com serviços de alta complexidade como internações e outros.

Pesquisas com amostragens populacionais de diferentes estados brasileiros apontam para aquisições relativamente baixas das medicações para DCNT como HAS E DM, demonstrando falhas na assistência farmacêutica da ESF. Gestão qualificada para o fornecimento medicamentoso tem suma importância. Fatores como funcionamento da Unidade de Saúde da Família (USF) apenas em horário comercial, nível socioeconômico da população, necessidade de medicação não disponível pelo sistema único de saúde (SUS) entram nesse cenário como relevantes no acesso medicamentoso e sua aderência. Conhecer o perfil populacional da comunidade local é crucial para melhor adequação do funcionamento e cobertura ampla do tratamento de DCNT (Vosgerau et al, 2011)

Dados coletados na Pesquisa Nacional sobre Acesso, Utilização e Promoção do Uso Racional de Medicamentos (PNAUM), coletados em 2013-2014 no Brasil, mostram um perfil de aquisição medicamentoso maior para DCNT em adultos e idosos. Além disso, apontam o SUS como a maior fonte de obtenção medicamentosa. Uma outra parcela da população - principalmente Nordeste e Norte do país - não é SUS exclusiva e busca farmácias privadas, indicando déficits 
de acesso dentro do próprio SUS. Por via SUS todas as medicações são gratuitas e destinadas às doenças crônicas de maior prevalência. Na Farmácia Popular o acervo de medicamentos é menor e o paciente adquire mediante copagamento, pois apenas alguns são gratuitos, principalmente para asma, diabetes e hipertensão.

A assistência farmacêutica e gestão de serviços de saúde se relacionam com o abastecimento das farmácias bem como controle do estoque medicamentoso disponível, a dissolução de possíveis barreiras burocráticas que fazem com que o paciente não consiga fazer aquisição na UBS e precise custeá-lo do próprio bolso. Além disso, o acesso depende também de um financiamento adequado (Matta SR et al, 2018).

Cabe às diferentes esferas governamentais o papel de garantir a qualidade no controle do percurso feito pela medicação até o paciente. A Política Nacional de Medicamentos faz atribuições às três esferas de governo e grande parte do controle medicamentoso aqui mencionado é de responsabilidade estatal, incluindo a formulação, execução, acompanhamento e avaliação da política de insumos e equipamentos direcionados a saúde.

Alguns autores consideram além do acesso a questão da qualidade das substâncias farmacológicas que constituem enalapril e propranolol por exemplo. Nessa avaliação de qualidade foram identificados desvios como irregularidade no teor do princípio ativo, peso médio, aspecto visual e friabilidade em quantidade de lotes considerável (Vosgerau et al, 2011).

\subsubsection{Diabetes mellitus}

Além de ser fator de risco para HAS a diabetes entra no grupo de DCNT relacionadas com DCV. Assim sendo, a adesão ao seu tratamento é também de grande importância na prevenção de eventos cardiovasculares.

Segundo a International Diabetes Federation (IDF) e dados de 2019, a prevalência mundial de diabetes mellitus é de 8,8\%. Metade dessas pessoas não sabe que tem a doença. A mesma organização coloca o Brasil no quarto lugar no ranking mundial, com mais de 12 milhões de pessoas acometidas com diabetes.

$\mathrm{O}$ fato de a doença poder se comportar de maneira silenciosa, sendo assintomática no seu estágio inicial em alguns pacientes, acaba por contribuir com um contexto de subdiagnóstico ou diagnóstico mais tardio. Além disso, a Sociedade Brasileira de Diabetes elucida que as medicações podem ser alteradas dependendo da idade e dos níveis glicêmicos do paciente. O controle das taxas de glicemia é obtido por meio de injeções de insulina e alguns pacientes necessitam receber a substância concomitante ao uso de remédios (CONITEC 11.05.2019).

Das drogas disponíveis para DM no SUS incluem insulinas humana NPH e regular, e os remédios metformina, glibenclamida e gliclazida. Mais recentemente foi acrescentada a dapagliflozina, pertencente a classe medicamentosa diferente das já ofertadas. Em análises de estudos verificou-se benefícios além da queda da glicose, acrescentando a redução da possibilidade de eventos cardiovasculares como infarto e outros, em pacientes de risco. A medida de trazer essa tecnologia alcança pacientes de DM 2, acima dos 65 anos, com DCV pre-estabelecida e que não obteve controle suficiente com os recursos usados e disponíveis pelo SUS até então. (CONITEC 2020).

\subsubsection{Dislipidemia}

Dislipidemia refere a modificações dos níveis lipídicos na circulação sanguínea, de ordem genética (primários) e por doenças, medicamentos, hábitos de vida e outros (secundários), e seu maior impacto nas DCV está relacionado às hiperlipidemias. Estas contribuem para formação de placa aterosclerótica por meio de formação de trombos oclusores nas artérias ou ainda por interromper o fluxo de sangue e ocasionar morte dos tecidos. As estatinas têm grande impacto na prevenção primária e secundária de DCV, atuando no perfil lipídico e ainda apresentando efeitos pleiotrópicos como inibição da proliferação de células do músculo liso e migração induzida por fatores de crescimento, influenciando na estabilidade das 
placas e formação de trombo. Também aumentam a disponibilidade de óxido nítrico e por consequência intervém na aterosclerose (Ana Paula Zambuzi Cardoso et al, 2021).

A sinvastatina é um medicamento genérico e por isso se tornou bem acessível. O maior problema do tratamento com estatinas é o custo, porém, quando este é calculado com a diminuição de eventos cardíacos, mortes prematuras, menor perda do tempo ativo de vida, a relação custo-benefício corrobora o investimento nas prevenções primária e secundária pelos gestores de saúde.

\subsection{Terapêutica não farmacológica}

Modificação do estilo de vida, principalmente práticas de exercício físico e alimentares, são muitas vezes primeiro passo nos tratamentos e mantém importância mesmo associados a farmacoterapia. Além disso, o controle de hábitos como tabagismo compõe significativamente esse campo. Por sua efetividade, as medidas de alteração no estilo de vida são benéficas para a economia em saúde.

\subsubsection{Alimentação}

A política Nacional de Alimentação e Nutrição, no Brasil, instituem diretrizes para promover alimentação saudável, limitando a ingesta de colesterol e sódio, por exemplo. Dietas ricas em fibras, alimentos de origem vegetal, quantidade pequena de carne vermelha, preferência por gorduras insaturadas, restrição alcoólica são medidas de controle para pessoas com risco de DCV e também proveitosas por grupos sem risco por auxiliar no controle do IMC (Silva et al, Análise de metanálises e ensaios clínicos relativos à utilização de estatinas, 2016).

Alguns estudos transversais realizados apontam uma ligação entre a renda e a disponibilidade de alimentos na família. O nível de renda familiar também é um indicador da qualidade de vida (Braga; Lopes, 2009). Não apenas a renda, mas também o apoio familiar influencia nos hábitos de vida. Muitas pessoas sentem dificuldade de mudança alimentar pela discrepância entre a dieta adotada e a dos demais membros familiares. Nesse sentido, nota-se necessidade de colaboração da família como sistema de relações integrativas que se ajuda. No entanto, importa ressaltar que cultura, sociedade e localização moldam significativamente o hábito familiar. Existem regiões com elevada ingesta de açúcar devido à quantidade e tipos de frutas disponíveis facilmente, por exemplo.

Além disso, o conhecimento acerca da própria doença é ferramenta para incentivo ao autocuidado. A compreensão sobre a patologia integra fator de adesão importante.

A baixa condição socioeconômica, a falta de conhecimento e a estrutura familiar compõem os principais desafios enfrentados por esses pacientes para a adoção de uma dieta balenceada. (Bastos, et al., 2020)

\subsubsection{Atividade física}

Praticar exercícios físicos regularmente contribui tanto para prevenção de doenças crônicas como constitui tratamento não farmacológico de agravos já existentes, ajudando a melhorar significativamente a qualidade de vida.

Assim como na dieta, na atividade física os fatores comportamentais, emocionais, familiares, de suporte e afins são preponderantes na adesão da prática disciplinada da recomendação como tratamento e/ou prevenção. No Brasil, estratégias para melhor emprego do autocuidado nas doenças crônicas já são desenvolvidas, como o Plano de Ações estratégicas para o enfrentamento de doenças crônicas, criado em 2011 e é válido até 2022.

A limitação de conceituar a exercício físico como a repetição de séries de movimentos corporais no intuito de melhorar a aptidão física advém de vertente militar, quando os treinos tinham uma finalidade também restrita. No entanto, se ampliamos o olhar para o termo "prática corporal" temos a ideia de atividades físicas abrangendo um dinamismo corporal que 
pode representar cultura, arte, envolvendo dança, jogos, esporte e outros. Fica claro que a prática corporal permite experiências, vivências, trocas, e trabalhos que vão além da queima de calorias e fortalecimento de musculatura. Isso traz o indivíduo para um contato mais harmonioso e produtivo com o tipo de atividade física exercida. Considerar o paciente com suas subjetividades permite adentrar melhor nas estratégias para adesão desse tratamento. Levando em conta esse aspecto, Ministério da Saúde aborda como necessário:

...reforçar que a mudança de hábitos é algo complexo e que o profissional precisa utilizar estratégias que facilitem esse processo, conhecer e utilizar os recursos disponíveis na comunidade, como os centros de convivência, os espaços de esporte e de lazer e as escolas. Espaços como os Polos de Academia da Saúde, espaços públicos construídos para a orientação e desenvolvimento de atividade física/práticas corporais, além de ações de segurança alimentar e nutricional, de educação alimentar e de práticas artísticas e culturais, podem ser oportunizados nesse sentido. A orientação dos profissionais de Saúde visando facilitar a escolha dos usuários acerca das atividades físicas/práticas corporais precisa levar em consideração hábitos de vida, preferências pessoais, assim como condições socioeconômicas individuais e comunitárias, práticas culturais, reforçando-se ainda o teatro, a música, a dança, a hidroginástica e as práticas da Medicina Tradicional Chinesa (tai chi chuan, lian gong, chi gong)

(MINISTÉRIO DA SAÚDE, 2012, ESTRATÉGIAS PARA O CUIDADO DA PESSOA COM DOENÇA CRÔNICA, Cadernos de Atenção Básica, p.80)

Os efeitos positivos no metabolismo de lipídios e da glicose, na pressão arterial, na densidade mineral óssea, na produção de hormônios e antioxidantes, no trânsito intestinal e nas funções psicológicas sugerem ser os principais mecanismos pelos quais a atividade física protege os indivíduos contra as doenças crônicas (Pederson; Saltin, 2006, por Ministério Da Saúde, 2014, Estratégias Para O Cuidado Da Pessoa Com Doença Crônica, Cadernos de Atenção Básica, p. 77)

\subsubsection{Tabagismo}

A abordagem do tabagismo envolve fortemente fator psicológico e comportamental, pois se associa, além da dependência química de substâncias, a hábitos, tensões e/ou momentos recreativos do paciente fumante. Desse modo, a maneira de abordagem com menção dos danos à saúde causados pelo tabaco, investigação do conhecimento do paciente acerca da própria dependência, influencia na adesão ao tratamento.

A terapia de reposição nicotínica é primeira escolha para abordagem do fumante. A substância pode ser reposta via goma de mascar, pastilha e adesivo na pele. A outra forma terapêutica utiliza bupropiona, que simula os efeitos nicotínicos no cérebro e pode ser feita em associação ao adesivo de nicotina ou não.

A parte mais dificultosa aparenta ser adentrar, convencer e interagir positivamente com a consciência do fumante no sentido de caminhar na adesão terapêutica rumo a cessação do tabagismo.

A avaliação completa acerca de dependência, abstinência e outros pontos que permeiam o hábito de fumar é muito importante, ou seja, aplicação da escala de Fangerstrom - que estima o grau de dependência à nicotina - associada à anamnese detalhada do paciente são de emprego efetivo para conduzir o tratamento e ajudar a lidar inclusive com fases terapêuticas de manutenção e outros períodos críticos como recaídas. Os estágios de motivação auxiliam no entendimento da fase em que a pessoa se encontra em relação ao tabagismo: Pré-contemplativo, contemplativo, determinado, ação, manutenção ou recaída. Tais motivações se relacionam com técnicas específicas de aconselhamento, que por sua efetividade denotam relevância do fator comportamental na aderência terapêutica. É possível ainda reforçar o suporte psicológico com terapia, principalmente cognitivo comportamental, bastante usada nesse manejo (Oliveira et al, 2019). 
A completude da anamnese contribui também para conhecimento de comorbidades como hipertensão arterial, dislipidemia, obesidade e outras que associadas ao tabagismo ampliam risco de DCV. Há necessidade de acessar esse conhecimento como ferramenta de estabelecimento de metas em conjunto com o paciente.

Países implementaram as medidas MPOWER lançadas pela OMS em 2007 para promover ações governamentais na luta contra o tabaco e contribuir para a preservação de vidas e prevenção de despesas com gastos em cuidados de saúde. Em relatório de 26 de julho de 2019, o Brasil foi o segundo país a alcançar alto nível de todas as intervenções da MPOWER, sendo elas:

- Monitoring: Monitorar uso de tabaco e políticas de prevenção

- Protecting: Proteger a população contra a fumaça do tabaco

- Offering: Oferecer ajuda para cessação do fumo

- Warning: Advertir sobre os perigos do tabaco

- Enforcing: Fazer cumprir as proibições sobre publicidade, promoção e patrocínio

- Raising: Aumentar os impostos sobre o tabaco

\section{Conclusão}

Adesão ao tratamento é o grau de concordância entre o comportamento de uma pessoa e as orientações do profissional de saúde. Dessa definição colhe-se que há uma série de variáveis que interagem e contribuem para baixa adesão a tratamentos medicamentosos bem como não farmacológicos (Mengue et al, 2014).

Em países como o Brasil, o destrinchar dos processos que envolvem adesão terapêutica em pacientes usuários de saúde pública, toca as desigualdades sociais, o déficit educacional e as significativas falhas de gestão em diferentes instâncias. É notória a indispensabilidade de gestores capacitados nas diversas esferas de governo e ainda na administração de setores da saúde do país, responsáveis com as finanças estatais, seus devidos direcionamentos e preocupados com o a redução do número de mortalidades da população prevenindo suas principais causas. Além disso, há necessidade de maior investimento em pesquisas científicas quantitativas que forneçam mais dados a respeito da adesão terapêutica e suas peculiaridades. O estímulo a educação em saúde e conhecimento dessas informações é fundamental para o desenvolvimento em saúde e para melhorias econômicas e sociais.

\section{Referências}

Almeida, E. R., Moutinho, C. B., \& Leite, M. T. S. A prática da educação em saúde na percepção dos usuários hipertensos e diabéticos Saúde Debate .|38, 328-337, 2014.

Alves, M. H., \& Pontelli, B. Doenças crônicas e a prática da atividade física no impacto das internações por causas sensíveis a atenção básica. Revista Fafibe On-Line, Bebedouro SP, 8 (1): 310-318, 2015.

Cardoso, A. P. Z., Nogueira, M. S., \& Hayashida, M. Aspectos clínicos e socioeconômicos das dislipidemias em portadores de doenças cardiovasculares, Physis Revista de Saúde Coletiva, 21 417-436, 2011

Araujo, A. P. V et. Al, Avaliação da eficácia da atenção farmacêutica no tratamento das dislipidemias, Revista Acadêmica Conecta FASF 2(1):26-34, 2017

Bastos, M. N. et al, Alimentação de diabéticos e hipertensos: desafios e recomendações, Educação em Saúde. Revista Revise, 4, (2020): O Sistema Único de Saúde na Formação e na Prática Médica, p. 173-191

Costa, J. A et al, Promoção da saúde e diabetes: discutindo a adesão e a motivação de indivíduos diabéticos participantes de programas de saúde Ciência \& Saúde Coletiva, 16(3):2001-2009, 2011

Giotto E. et al, Adesão ao tratamento farmacológico e não farmacológico e fatores associados na atenção primária da hipertensão arterial, Ciênc. saúde coletiva, 2013

Oliveira, G. M. M et al, Recomendações de 2019 para redução do consumo de tabaco nos países de língua portuguesa, Revista Portuguesa de Cardiologia, Perspetivas Em Cardiologia, 2019;38(4): 233-244 
Research, Society and Development, v. 10, n. 7, e39210716778, 2021

(CC BY 4.0) | ISSN 2525-3409 | DOI: http://dx.doi.org/10.33448/rsd-v10i7.16778

Gomes-Villas Boas, L. C. et al, Relação entre apoio social, adesão aos tratamentos e controle metabólico de pessoas com diabetes mellitus. Revista Latino Americana de Enfermagem, 20, 2012

Ministério Da Saúde, Estratégias para o cuidado da pessoa com doença crônica, 2014. http://189.28.128.100/dab/docs/port aldab/publicacoes/caderno_35.pdf

Núcleo de Telessaúde do Rio Grande do Sul, A sinvastatina é a estatina mais adequada a ser empregada no sus? BVS Atenção Primária em Saúde, Cuidados Primários de Saúde, 2010, https://aps.bvs.br/aps/a-sinvastatina-e-a-estatina-mais-adequada-a-ser-empregada-no-setor-publico-sus/

OPAS (Organização Pan-americana de Saúde), OMS lança novo relatório sobre a epidemia mundial do tabaco; Brasil é $2^{\circ}$ país a alcançar alto nível de todas as medidas MPOWER. Determinantes Sociais e Riscos para a Saúde, Doenças Crônicas Não Transmissíveis e Saúde Mental, https://www.paho.org/bra/index.php?option=com_content\&view=article\&id=5993:oms-lanca-novo-relatorio-sobre-a-epidemia-mundial-do-tabaco-brasil-e-2pais-a-alcancar-alto-nivel-de-todas-as-medidas-mpower \&Itemid=839

Ribeiro et al, Tratamento não farmacológico da hipertensão na atenção primária à saúde: ensaio clínico comparativo de duas estratégias de educação em saúde e nutrição, BMC Public Health 2011, 11:637

SR, Matta, Fontes de obtenção de medicamentos por pacientes diagnosticados com doenças crônicas, usuários do Sistema Único de Saúde

Silva HL, et al, Análise de metanálises e ensaios clínicos relativos à utilização de estatinas, Rev Pan-Amaz Saude 2016; 7(4):107-119 1

Tavares, N. U. L. et al, Acesso e uso de medicamentos para hipertensão arterial no Brasil, Revista Saúde Pública 2016;50(supl 2):8s Tavares NUL et al.,

Tavares, N. U. L. et al, Acesso gratuito a medicamentos para tratamento de doenças crônicas no Brasil, Revista Saúde Pública 2016;50(supl 2):7s

Tavares, N. U. L. et al, Fatores associados a baixa adesão ao tratamento farmacológico de doenças crônicas no Brasil, Revista Saúde Pública 2016;50(supl 2): $10 \mathrm{~s}$

Vosgerau, et al, Utilização de anti-hipertensivos e antidiabéticos, Rev Bras Cardiol. 2011;24(2):95-104 março/abril 